\title{
In vitro-in vivo correlation from lactide-co-glycolide polymeric dosage forms
}

\author{
Susan D'Souza • Jabar A. Faraj · Stefano Giovagnoli • \\ Patrick P. DeLuca
}

Received: 4 September 2014/Accepted: 25 September 2014/Published online: 2 December 2014

(C) The Author(s) 2014. This article is published with open access at Springerlink.com

\begin{abstract}
The objective of this study was to compare the in vitro behavior of four long-acting subcutaneous risperidone formulations with in vivo performance, with the intent of establishing an IVIVC. Two copolymers of PLGA (50:50 and 75:25) were used to prepare four microsphere formulations of risperidone, an atypical antipsychotic. In vitro behavior was assessed at the physiological temperature $\left(37^{\circ} \mathrm{C}\right)$ using the 'modified dialysis' technique. The in vitro release profile demonstrated rank order behavior with Formulations $A$ and $B$, prepared using the 50:50 copolymer, exhibiting rapid drug release, while Formulations $C$ and $D$, prepared using
\end{abstract}

S. D'Souza $(\bowtie)$ · J. A. Faraj · S. Giovagnoli · P. P. DeLuca University of Kentucky College of Pharmacy, Lexington, KY 40536, USA

e-mail: dr_ssdsouza@yahoo.com

P. P. DeLuca

e-mail: patrick.deluca@uky.edu

Present Address:

S. D'Souza

Sunovion Pharmaceuticals Inc, Marlborough,

MA 01752, USA

Present Address:

J. A. Faraj

Evonik Inc, 750 Lakeshore Parkway, Birmingham,

AL 35211, USA

e-mail: jabarfaraj2002@yahoo.com

Present Address:

S. Giovagnoli

Department of Chemistry and Technology of Drugs,

Università degli Studi di Perugia, Via del Liceo 1,

06123 Perugia, Italy

e-mail: stefano.giovagnoli@unipg.it
75:25 PLGA, released drug in a slower manner. In vivo profiles were obtained by two approaches, i.e., deconvolution using the Nelson-Wagner equation (the FDA recommended approach) and using fractional AUC. With both in vivo approaches, the 50:50 PLGA preparations released drug faster than the 75:25 PLGA microspheres, exhibiting the same rank order observed in vitro. Additionally, profiles for the four formulations obtained using the deconvolution approach were nearly superimposable with fractional AUC, implying that the latter procedure could be used as a substitute for the Nelson-Wagner method. A comparison of drug release profiles for the four formulations revealed that in three of the four formulations, in vivo release was slightly faster than that in vitro, but the results were not statistically significant $(P>0.0001)$. An excellent linear correlation $\left(R^{2}\right.$ values between 0.97 and 0.99 ) was obtained when $\%$ in vitro release for each formulation was compared with its corresponding in vivo release profile, obtained by using fraction absorbed (Nelson-Wagner method) or fractional AUC. In summary, using the four formulations that exhibited different release rates, a Level A IVIVC was established using the FDA-recommended deconvolution method and fractional AUC approach. The excellent relationship between in vitro drug release and the amount of drug absorbed in vivo in this study was corroborated by the nearly $1: 1$ correlation $\left(R^{2}\right.$ greater than 0.97$)$ between in vitro release and in vivo performance. Thus, the results of the current study suggest that proper selection of an in vitro method to assess drug release from long-acting injectables will aid in obtaining a Level A IVIVC.

Keywords Risperidone - PLGA microspheres - In vitroin vivo correlation - Level A - Modified dialysis method . Nelson-Wagner $\cdot$ Fractional AUC 


\section{Background}

The delivery of therapeutic agents using controlled release polymers has been an area of research that has witnessed extensive growth. Indeed, the nature and properties of these polymers lend themselves well to the design and development of complex dosage forms, whether for administration via the oral or non-oral route. One such polymer that has consistently demonstrated success with controlled release injectables is the PLGA (polylactide-co-glycolide) copolymer (Schrier and DeLuca 1999; Hora et al. 1990; Mehta et al. 1994; Capan et al. 1999). The PLGA polymer is biodegradable, biocompatible and has been approved for human use by the United States Food and Drug Administration (US FDA) as surgical sutures, implantable devices and drug delivery systems (D'Souza et al. 2013c; Middleton and Tipton 2000; He et al. 2011; Wei et al. 2004). Formulation of an injectable with a versatile polymer like PLGA provides significant benefits. For instance, PLGAbased formulations such as biodegradable microspheres are able to bypass the GI tract and allow enhanced bioavailability of molecules with a short half-life. e.g., peptides and proteins. Other advantages include reduced frequency of dosing, improved patient compliance, maintenance of consistent blood levels and less systemic side effects due to controlled delivery of the therapeutic agent upon intramuscular or subcutaneous administration (D'Souza et al. 2013a; Xuan et al. 2013; Shmueli et al. 2013; Kwak et al. 2010). Thus, the long history of safety and advantages of the PLGA polymer have resulted in several commercialized formulations and billions of dollars in revenue (Chaubal 2002).

Several physical and chemical polymer properties regulate drug release from the PLGA polymer. These include molecular weight, copolymer composition, crystallinity and hydrophilicity (DeLuca et al. 1993; Park et al. 2007; Xuan et al. 2013; Sun et al. 2009). A careful selection of polymer and dosage form properties ensures customized drug release for varying duration of action, ranging from days to several months, as evidenced by numerous reports (Ertl et al. 1999; Woo et al. 2001). Hence, monitoring drug release from PLGA-based formulations, both in vivo and in vitro, routinely requires extended study periods. In vivo measurements of drug release from injectable dosage forms, though preferred, entail considerable time to plan and conduct, are expensive and labor-intensive. In contrast, in vitro studies, a surrogate for in vivo assessments, are much simpler to perform and provide extensive insight into the rate and mechanism of drug release (D'Souza et al. 2014a; Washington 1990). Therefore, investigations of in vitro-in vivo correlations (IVIVC) between in vitro drug release and in vivo bioavailability are progressively becoming central to the development of extended-release products (Uppoor 2001; Siewert et al. 2003; Martinez et al. 2008). However, when compared with conventional dosage forms, literature on IVIVC with extended-release injectables continues to remain sparse.

For an IVIVC, it is critical to select the appropriate in vitro conditions such that the in vivo environment is mimicked as closely as possible without any change in the release mechanism(s). While in vitro evaluation of dosage forms like oral tablets or capsules is straightforward because compendial apparatus can be utilized, measurement of drug release from non-oral dosage forms like injectable microspheres is not as simple. Lack of a compendial apparatus has led to a proliferation of apparatus that are used to assess drug release (D'Souza and DeLuca 2006). For this reason, several methods have been developed to study drug release from injectable microspheres.

A literature survey reveals that in vitro drug release methods for injectable dosage forms such as PLGA microspheres fall into three categories. The most popular 'sample and separate' or 'tube' method involves introduction of the injectable dosage form into a container containing release media, and drug release is assessed over time by filtration [analysis of the filtrate (Yen et al. 2001)] or centrifugation [analysis of the supernatant (Park et al. 1998) or remaining drug (Blanco-Prieto et al. 2004)]. The 'tube' method, though simple to set up and use, has significant drawbacks. Lower drug release rates have been reported and are a consequence of aggregation of microspheres in the release media (Bain et al. 1999). Sampling and buffer replacement during the later stages of drug release are quite cumbersome due to the formation of small-sized particles (by-products of polymer degradation) causing filter clogging. Further, sink conditions are difficult to maintain due to challenges involved with partial or total buffer replacement of the release media for the extended duration of the in vitro release study. Nevertheless, IVIVCs with biodegradable microspheres have been attempted using the 'sample and separate' in vitro method, with varying degree of success (Jiang et al. 2002; Morita et al. 2001; Heya et al. 1994).

The 'continuous flow' method (an adaptation of USP apparatus 4), is another technique that has been used to study in vitro drug release from injectable microspheres (Aubert-Pouessel et al. 2002). In the 'continuous flow' method, the release media is circulated or re-circulated (via a pump) through a column containing injectable microspheres. Sampling of the release media at pre-determined intervals allows for an evaluation of drug release over a period of time. Several types of pumps have been reported including syringe pumps (Aubert-Pouessel et al. 2002, 2004), HPLC pumps (Longo and Goldberg 1985) and peristaltic pumps (Wagenaar and Muller 1994) allowing for a wide range of flow rates as low as $5 \mu \mathrm{L} / \mathrm{min}$ to a high 
200 L/h (D'Souza and DeLuca 2006). Though the setup of the 'continuous flow' method permits convenient sampling followed by buffer replacement, major shortcomings have been reported. Constant flow rates are difficult to achieve in the later stages of polymer degradation. Indeed, polymer breakdown causes filter clogging and has been reported to result in high pressure buildup leading to variable flow rates. Other disadvantages include cumbersome setup, static charge issues with glass beads and challenges with rapid buffer replacement (partial or total) (Rawat et al. 2011). These challenges make it difficult to establish an IVIVC using the 'continuous flow' method.

A third method utilizing the 'dialysis' principle has also been reported in literature (Kostanski and DeLuca 2000; D'Souza et al. 2014a). In this method, the injectable microspheres are placed into a dialyzer containing a small volume of release media which in turn is introduced into a container contain a larger volume of release media (outer bulk). As the polymer degrades and drug is released, it diffuses out of the dialyzer into the outer bulk, from where it is sampled. This physical separation of the injectable microspheres from the outer bulk media eliminates filter clogging issues reported with the 'tube' and 'continuous flow' methods. A key advantage of the 'dialysis' method is that it mimics in vivo conditions where the injectable microspheres are immobilized upon subcutaneous or intramuscular administration and surrounded by a stagnant layer causing slow diffusion of drug since sink conditions are not maintained (Nastruzzi et al. 1993). Setup issues with older systems such as dialysis bags (Diaz et al. 1999) have been eliminated with the introduction of commercially available dialyzers (Woo et al. 2001; D'Souza et al. 2014a). Of the three methods, partial or total buffer replacement of the outer bulk is straightforward, allowing sink conditions to be maintained throughout the duration of the in vitro study. The simplicity of this method lends itself well to an evaluation of in vitro release. Indeed, published results have confirmed the utility of this method in establishing an IVIVC with biodegradable microspheres (D'Souza et al. 2014b; Kostanski et al. 2000).

Given the advantages of the 'dialysis' method over the 'sample and separate' and 'continuous flow' methods, its suitability for use in the development of an IVIVC is obvious. Therefore, the objective of this study was to develop an IVIVC using the 'dialysis'-based technique for previously developed PLGA-based microsphere preparations of risperidone (D'Souza et al. 2013b). Of the different 'dialysis'-based techniques, the 'modified dialysis' method was selected to evaluate in vitro release. Further, an IVIVC was attempted using two approaches, deconvolution and fractional AUC.

\section{Materials and methods}

Materials

Risperidone was purchased from Cipla Ltd., India, and PLGA 50:50 (45 and 74 kDa) and 75:25 (54 and $65 \mathrm{kDa})$ from Boehringer Ingelheim (Ingelheim, Germany) and Alkermes (Cambridge, MA). All other chemicals were obtained commercially as analytical-grade reagents.

Preparation of microspheres

Risperidone PLGA microspheres were prepared by a solvent extraction/evaporation method, as described previously (D'Souza et al. 2013b). Briefly, a solution of drug and polymer was injected into an aqueous continuous phase under stirring with a Silverson L4R mixer (Silverson machines, MA, USA) at a pre-determined speed. The solvents were removed by stirring for $2 \mathrm{~h}$ at $40{ }^{\circ} \mathrm{C}$. The resulting microspheres were recovered by filtration, washed and freeze dried in unit vials along with the diluent. Briefly, the four formulations prepared were:

(a) 45 kDa PLGA, 50:50 lactide:glycolide (Formulation A),

(b) $74 \mathrm{kDa}$ PLGA, 50:50 lactide:glycolide (Formulation $B)$,

(c) $54 \mathrm{kDa}$ PLGA, 75:25 lactide:glycolide (Formulation C) and

(d) $65 \mathrm{kDa}$ PLGA, 75:25 lactide:glycolide (Formulation $D)$.

Drug content

Drug content and encapsulation efficiency were determined for all the formulations. Briefly, 5-10 mg of microspheres was dissolved in $10 \mathrm{~mL}$ of acetonitrile; $40 \mathrm{~mL} 0.1 \mathrm{M}$ acetate buffer, $\mathrm{pH} 4.0$, added and the solution gently mixed. The solution was filtered through a PTFE syringe filter prior to analysis by HPLC. The analysis was performed by injecting $50 \mu \mathrm{L}$ samples in a HPLC C-18 column in gradient mode. The mobile phases were (A) $0.1 \%$ TFA aqueous solution; (B) acetonitrile with $0.1 \%$ TFA. The gradient method was: $80 \% \mathrm{~A}, 20 \% \mathrm{~B}$ to $50 \% \mathrm{~A}$, $50 \% \mathrm{~B}$ over $12 \mathrm{~min}$ at a flow rate of $1.5 \mathrm{~mL} / \mathrm{min}$. Measurements were made in triplicate. Drug content for Formulations $A-D$ was determined to be $25,34,34$ and $33 \%$, respectively (D'Souza et al. 2013b).

In vitro release

The in vitro study was performed in $0.1 \mathrm{M}$ phosphate buffered saline, $\mathrm{pH} 7.4$, containing $0.05 \%$ Tween- $80^{\circledR}$ and 
$0.1 \%$ sodium azide using a 'modified dialysis' method (Kostanski and DeLuca 2000; D'Souza et al. 2014b). Risperidone PLGA microspheres were accurately weighed and placed in a 7-mL dialysis tube (Tube-ODilalyzer $^{\circledR}$, MWCO $300,000 \mathrm{Da}$ ) filled with $5.0 \mathrm{~mL}$ of release media, which in turn was placed in a $50-\mathrm{mL}$ tube containing $40 \mathrm{~mL}$ of the same release medium (outer bulk). The contents of the larger tube were continuously stirred with a magnetic stirrer. All tubes were incubated at $37^{\circ} \mathrm{C}$. At each time point $1.0 \mathrm{~mL}$ was removed from the $50-\mathrm{mL}$ tube (outer bulk) and $1.0 \mathrm{~mL}$ of fresh buffer was added. Risperidone content was determined by HPLC.

In vivo study

Male Sprague-Dawley rats $(n=6)$ weighing $\sim 300 \mathrm{~g}$ were used to evaluate the in vivo performance of risperidone microspheres. The microspheres were injected subcutaneously at the back of the neck (20-40 mg/kg dose of risperidone/rat) after reconstitution in a suitable vehicle containing sodium carboxymethylcellulose, mannitol and Tween $80^{\circledR}$. Blood samples were collected from the tail vein at specific time points and centrifuged in Microtainer ${ }^{\circledR}$ tubes (Becton-Dickinson, Franklin Lakes, NJ) to collect the serum. Serum samples were frozen and stored at $-20{ }^{\circ} \mathrm{C}$ until analysis by an outside laboratory, Medtox Laboratories, MN.

\section{Development of an IVIVC}

Currently, there is no FDA guidance on establishing IVIVC for injectable dosage forms. However, using the FDA document for solid oral dosage forms, IVIVC relationships can be extrapolated to include non-solid oral delivery systems (FDA guidance for industry: extended-release oral dosage forms: development, evaluation and application of in vitro/in vivo correlations 1997). Per the guidance, an IVIVC can be categorized as follows (Uppoor 2001; D'Souza et al. 2014b):

1. Level A correlation is a point to point correlation between in vitro dissolution and in vivo absorption. That is, the in vitro dissolution profiles are typically superimposable with in vivo absorption curves or may be made superimposable by use of an appropriate scaling factor.

2. Level B correlation describes a relationship between summary parameters such as in vitro dissolution rate and in vivo absorption rate (e.g., mean dissolution time, MDT, vs mean residence time, MRT). By its definition, it is not a point to point correlation as several in vivo curves can produce a similar MRT value or mean in vitro dissolution curve.

3. Level $\mathrm{C}$ correlation is a single point comparison of the amount dissolved in vitro at a particular time (e.g., $T_{50} \%$ ) and an in vivo pharmacokinetic parameter (e.g., area under the curve, AUC). It does not describe the nature of the in vivo release profile, which is an important aspect in the characterization of performance from extended-release drug products.

In the current study, an IVIVC for the four risperidone PLGA formulations was determined using two approaches.

a. Nelson-Wagner approach: Of the FDA-recommended approaches for developing an IVIVC, the NelsonWagner technique was selected as it is suitable for use in drugs that follow a one-compartment pharmacokinetic model. The fraction absorbed $\left(F_{\mathrm{abs}}\right)$ was determined from the plasma concentration-time data by deconvolution using the Nelson-Wagner method as described in Eq. 1 (Wagner and Nelson 1963).

$F_{\text {abs }}(t)=\left[C(t)+k_{\mathrm{e}} \times \mathrm{AUC}_{(0-t)}\right] /\left[\begin{array}{ll}k_{\mathrm{e}} \times \mathrm{AUC}_{(0-\mathrm{inf})} & \end{array}\right]$.

With the Nelson-Wagner equation, the pharmacokinetic profile is deconvoluted to obtain the in vivo absorption as a function of time and is plotted alongside the in vitro release data to assess the superimposability of the two profiles. If the two curves are superimposable and a linear relationship is obtained, it suggests a strong correlation between in vivo and in vitro drug release.

b. Fractional AUC approach: The area under the curve (AUC) was calculated using the trapezoidal rule (Eq. 2)

$\operatorname{AUC}\left(t_{1}-t_{2}\right)=\left[\left(C_{1}+C_{2}\right) / 2\right] \times\left(t_{2}-t_{1}\right)$.

The fractional AUC was determined by dividing cumulative AUC at time ' $\mathrm{t}$ ' with cumulative $\mathrm{AUC}_{(0-\text { last })}$, as described in previous publications (Woo et al. 2001; Chu et al. 2006; D'Souza et al. 2014b) and plotted along with the $\%$ drug released in vitro. In a manner similar to the Nelson-Wagner approach, the superimposability of the in vivo and in vitro drug release was compared.

\section{Results}

In vitro release

The in vitro release results for Formulations $A-D$ are shown in Fig. 1. These results were obtained using the 'modified dialysis' method. At first glance, it is evident that Formulations $A$ and $B$, prepared using the 50:50 copolymer, release drug much faster than Formulations $C$ and $D$ 


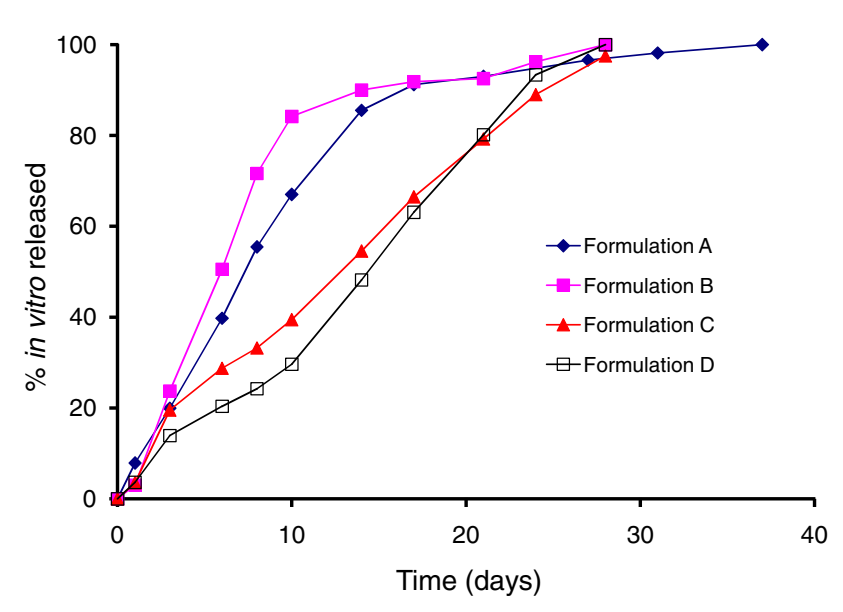

Fig. 1 In vitro release of risperidone PLGA microspheres (Formulations $A, B, C$ and $D$ )

that were manufactured using a copolymer with a $75: 25$ lactide:glycolide ratio. With all four formulations, a moderate initial burst was observed (day 1) after which drug release increased to reach between 15 and $20 \%$ by day 3 . After this, Formulations $A$ and $B$ maintained their release rate such that nearly $50 \%$ of the drug was depleted from the microspheres within a week. In contrast, the release rate for Formulations $C$ and $D$ rose in a more sustained fashion to realize $50 \%$ drug release in about 2 weeks, i.e., approximately twice the time observed with formulations containing the 50:50 copolymer. When approximately $85 \%$ drug was released from Formulations $A$ and $B$ (i.e., 2 weeks), the drug release rate slowed considerably till complete release was achieved. On the other hand, drug release from the 75:25 copolymer formulations remained consistently steady till $100 \%$ of the drug was released from the microspheres.

Literature cites that drug release from PLGA matrices such as biodegradable microspheres involves three phases (D'Souza et al. 2013a, 2014b). The first phase of release occurs immediately after the microsphere encounters a liquid medium (i.e., in vitro buffer or in vivo fluids). This phase of drug release is termed as 'initial burst' and is attributed to the release of drug that is bound to the surface of the microsphere or associated with easily accessible pores. The next two phases of drug release, i.e., diffusional and erosional release, are non-instantaneous and occur over a varying time course. During the diffusional phase of release, water intrusion leads to polymer hydration and slow movement of the encapsulated drug to the outer sink. The presence of water inside the polymer ensures hydrolysis of the polymeric ester bonds causing an autocatalytic effect leading to bulk hydrolysis. Rapid polymer degradation ensues, followed by erosion of the PLGA matrix and mass loss. Thus, drug release rates are much faster during the erosional phase. From Fig. 1, it is evident that all four formulations hydrated rapidly after which drug release rates rose till complete release was achieved.

The differences in vitro behavior of the four formulations can be explained on the basis of the lactide content of these microspheres. Previous studies have documented the differences in the in vitro release profiles between the 50:50 and 75:25 copolymers and ascribed it to a faster degradation rate in copolymers with lower lactide content, i.e., 50:50 PLGA will degrade much faster than the 75:25 copolymer (D'Souza et al. 2014b; Park 1995). Indeed, in comparison to the smaller glycolide species, greater amounts of the larger, more sterically hindered lactide moiety will reduce the degradation rate in a polymer.

Within the same copolymer, a comparison of release profiles for Formulations $A$ and $B$ (prepared using 50:50 PLGA) revealed slightly faster release for the higher molecular weight Formulation B (Fig. 1). This was presumably due to the higher drug load (i.e., higher drug to polymer ratio) and lower bulk density for Formulation $B$ (i.e., 0.67 vs. $0.76 \mathrm{~g} / \mathrm{cc}$ for Formulation A) (D'Souza et al. 2013b). Between Formulations $C$ and $D$, the former exhibited slightly faster drug release that could be attributed to its lower molecular weight.

Overall, the in vitro release experiments revealed the following:

(a) A moderate initial burst for all formulations;

(b) Faster drug release from the 50:50 copolymers; and

(c) Suitability of the 'modified dialysis' method in assessing the in vitro release from the four formulations.

\section{In vivo results}

In a previous study, we reported the preparation, characterization and in vivo evaluation of risperidone PLGA microspheres (D'Souza et al. 2013b). Formulations $A$ and $B$ were administered to rats at a $20 \mathrm{mg} / \mathrm{kg}$ dose, while Formulations $C$ and $D$ were administered at a $40 \mathrm{mg} / \mathrm{kg}$ dose. The in vivo release profiles for the four formulations are shown in Fig. 2.

\section{Fraction absorbed}

Figure 3 shows the fraction absorbed as a function of time for Formulations $A$ and $B(20 \mathrm{mg} / \mathrm{kg}$ dose $)$ and Formulations $C$ and $D(40 \mathrm{mg} / \mathrm{kg}$ dose $)$ in rats. The fraction of drug absorbed was calculated using the Nelson-Wagner method (described in Sect. "Development of an IVIVC"), i.e., by deconvoluting the pharmacokinetic profile to obtain the in vivo absorption as a function of time (Wagner and Nelson 1963). Deconvolution is a numerical method used to estimate the time course of drug input using a 
Fig. 2 In vivo release of risperidone from PLGA microspheres (Formulations $A$ and $B=20 \mathrm{mg}$ dose, and Formulations $C$ and $D=40 \mathrm{mg}$ dose) [data from ref. D'Souza et al. (2013b)]
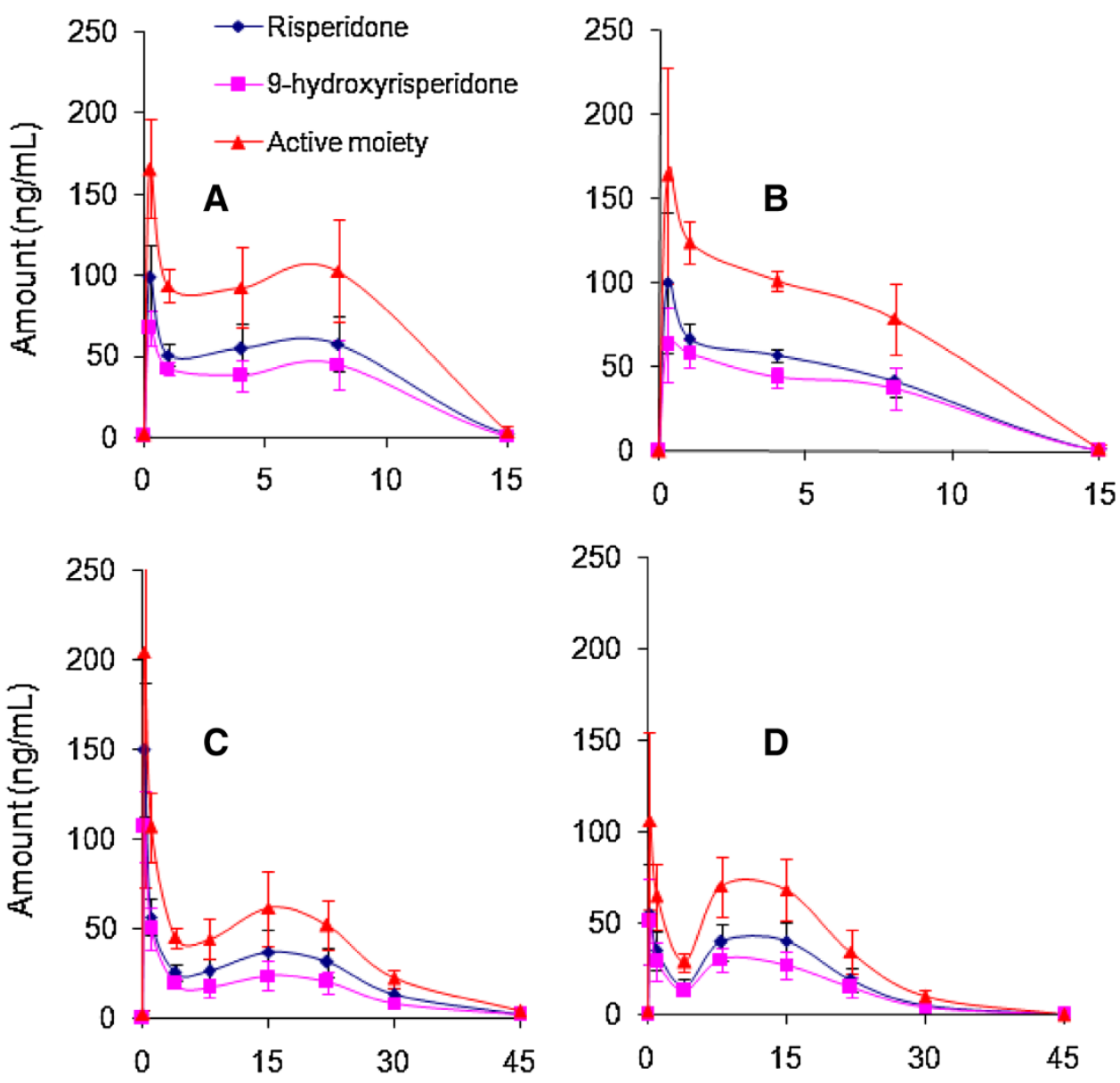

\section{Time (days)}

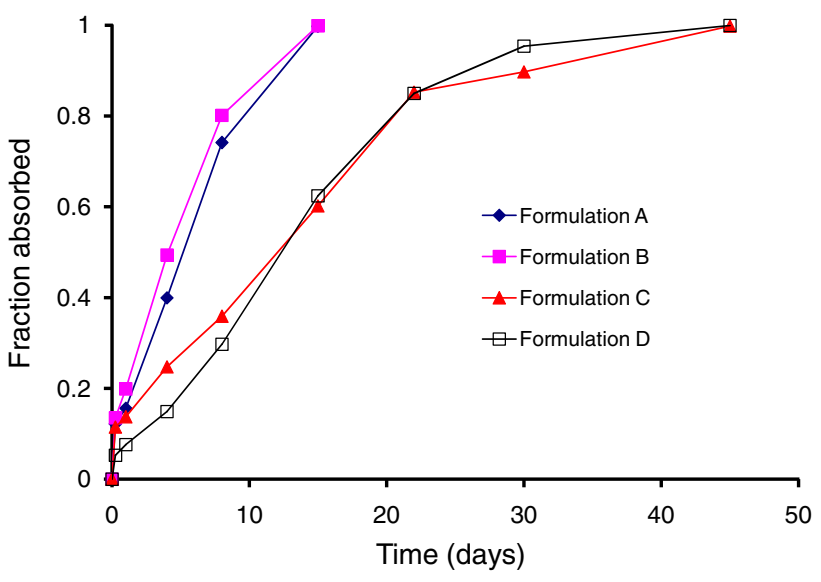

Fig. 3 Fraction of risperidone absorbed in vivo (Nelson-Wagner method)

mathematical function. After deconvolution, the in vivo and in vitro release profiles are plotted together to assess the superimposability of the two curves, allowing a direct comparison of release behavior.
A plot of the fraction of drug absorbed for the four formulations is shown in Fig. 3. In a manner similar to the in vitro release profiles described in Fig. 1, the differences in the fraction absorbed for the four formulations are easily discernible and a rank order is assigned. Of the four formulations, Formulations $A-C$ show a much larger initial burst than Formulation $D$. The initial burst value at day 1 for Formulation $D$ was around $7 \%$, nearly two to three times lower than the 13-20\% drug absorption seen with Formulations $A-C$. Between the copolymers, the 50:50 PLGA formulations (Formulations $A$ and $B$ ) demonstrate a rapid absorption profile, while Formulations $C$ and $D$ indicate a more sustained absorption curve. In fact, the absorption profiles including initial burst release for Formulations $A$ and $B$ are nearly identical, similar to the in vitro release profiles (Fig. 1) and serum data from rats (Fig. 2) with complete release achieved within 15 days. On the other hand, between the 75:25 polymers, Formulation $D$ exhibits slightly slower absorption profile through day 8 , after which the rate of absorption increases and is nearly the same as Formulation $C$. 
The differences in the fraction of active moiety absorbed from the 50:50 and 75:25 copolymers are clearly evident from day 4 where the value observed from Formulations $A$ and $B$ was nearly twice the amount noted with Formulations $C$ and $D$. This trend continued through day 15, when complete absorption was observed from formulations prepared with 50:50 PLGA. In contrast, the time taken for complete absorption for formulations prepared with the 75:25 copolymer was nearly thrice as long as with the 50:50 PLGA. Notably, however, the in vivo drug release profiles obtained by deconvolution (Nelson-Wagner method) were similar, but slightly faster than that observed under in vitro conditions, indicating that the mechanism of release was unchanged.

\section{Fractional AUC}

Figure 4 depicts a plot of the fractional AUC profile for the four formulations, calculated as described in Sect. "Development of an IVIVC". In a manner similar to the in vitro release profiles (Fig. 1), the fractional AUC profiles show rank order behavior. Akin to the fraction absorbed (Fig. 3), Formulation D had the lowest initial burst, nearly two to three times lower than the remaining formulations (Fig. 4). After the initial burst release of drug, fractional AUC profiles of Formulations $A$ and $B$, prepared using the 50:50 polymer, are essentially similar, with slight differences in the behavior observed between Formulations $C$ and $D$, prepared using the 75:25 polymer. Complete release was achieved in 15 days and 45 days for the 50:50 and 75:25 PLGA formulations, respectively, corroborating the release pattern observed in Fig. 3 (fraction absorbed).

As noted with the in vitro data (Fig. 1) and fraction absorbed (Fig. 3), the microspheres prepared from the lower lactide-containing copolymer exhibited a faster release rate than the high lactide-containing copolymer.

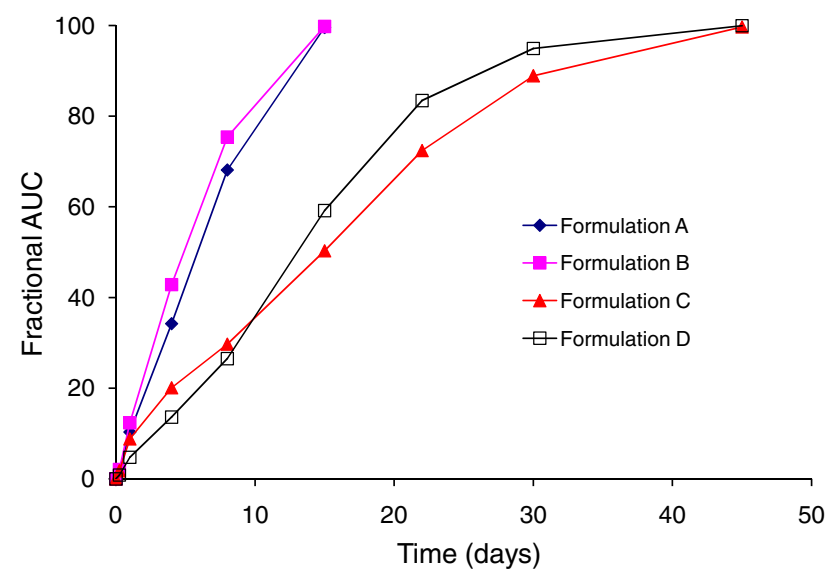

Fig. 4 Fractional AUC profile of risperidone from Formulations A, $B, C$ and $D$
The fractional AUC profiles for Formulations $A$ and $B$ are nearly identical throughout the time course of drug release. With Formulations $\mathrm{C}$ and D, Formulation $D$ exhibits slightly slower absorption profile through day 8 after which the rate of absorption increases and is slightly greater than Formulation $C$. Starting from day 4 , the differences in fractional AUC profiles between the 50:50 and 75:25 copolymers illustrate that the lower lactide-containing microspheres release drug twice as fast as the higher lactide-containing preparations. This behavior continued till complete release was attained with the 50:50 PLGA preparations. Similar to the profiles shown in Fig. 3 (fraction absorbed), the time taken for complete release with the 75:25 copolymer was threefold greater than the 50:50 PLGA polymer. Overall, the in vivo drug release profiles obtained using the fractional AUC approach were similar to that observed under in vitro conditions, indicating that the mechanism of release was unchanged.

\section{IVIVC}

From the FDA guidance for extended-release oral dosage forms, the most common approach for developing a Level A IVIVC encompasses the steps below:

(a) Develop formulations with different release rates (e.g., fast, intermediate, slow);

(b) Obtain in vitro and in vivo release profiles for the formulations; and

(c) Use an appropriate deconvolution technique (e.g., Nelson-Wagner) to calculate the in vivo absorption after which a correlation may be obtained by comparing in vivo behavior with in vitro release to establish an IVIVC (FDA guidance for industry: extended release oral dosage forms: development, evaluation and application of in vitro/in vivo correlations 1997).

The objective of an IVIVC is to establish a correlation between the in vitro dissolution behavior and in vivo performance of a drug product. Per the guidance, an IVIVC should be demonstrated with two or more formulations of different release rates and show corresponding differences in their absorption profiles. While three or more formulations with different release rates are recommended, an IVIVC may also be defined with a minimum of two or more formulations having different release rates (FDA guidance for industry: extended release oral dosage forms: development, evaluation and application of in vitro/in vivo correlations 1997). As such, an IVIVC is generally described by a linear relationship between parameters derived from the in vitro and in vivo experiments as quantified by the Pearson correlation. 
From a drug product perspective, establishing an IVIVC offers several benefits. As noted in literature, having an IVIVC allows in vitro release to be used as a surrogate for in vivo measurements from conventional or long-acting dosage forms (D'Souza and DeLuca 2006). Moreover, it reduces the time, labor and costs associated with performing bio-studies in humans or animals, while also minimizing unnecessary use of humans or animals for evaluation of drug release. For drugs that are in the late stages of development, IVIVCs can play an important role in characterizing process-related changes and also simplify any scale-up or post-approval changes, or to obtain a biowaiver. Finally, it ensures compliance with regulatory requirements and allows for clinically relevant in vitro dissolution specifications to be set (D'Souza et al. 2014b).

Based on the FDA guidance, a few publications have attempted to investigate an IVIVC from risperidone PLGA systems. For example, Su et al. (2009) investigated the in vivo behavior of several batches of risperidone microspheres formulated using uncapped and capped 75:25 PLGA. Using the 'sample and separate' method to assess in vitro drug release, a Level A IVIVC was obtained for a single batch of microspheres. In another publication, Amann et al. (2010) prepared risperidone implants with a series of PLGA polymers and obtained a good Level B correlation with the 'sample and separate' method. Given the advantages of the 'dialysis' method over the 'sample and separate' method, there is a strong rationale for using this technique to establish an IVIVC.

From the current study, Formulations $A$ and $B$ had the fastest release rate, while Formulation $C$ and $D$ had a slower release rate. Hence, all four formulations were deemed suitable for analysis of their release profiles with the goal of establishing an IVIVC. As described in Sect. "Development of an IVIVC", IVIVC was attempted by using two approaches. This type of data analysis wherein IVIVC was attempted and successfully achieved has been reported recently (D'Souza et al. 2014b). The first approach compares the in vitro release profiles to the fractional AUC curves, as reported previously by several authors (Woo et al. 2001; D'Souza et al. 2014b; Chu et al. 2006), while the second approach uses the FDA-recommended Nelson-Wagner method to calculate the absorption profile of the drug (FDA guidance for industry:
Fig. 5 Comparison of in vitro and in vivo release of Risperidone from PLGA microspheres (diamonds in vitro release, squares fractional AUC, triangle Nelson-Wagner absorption)
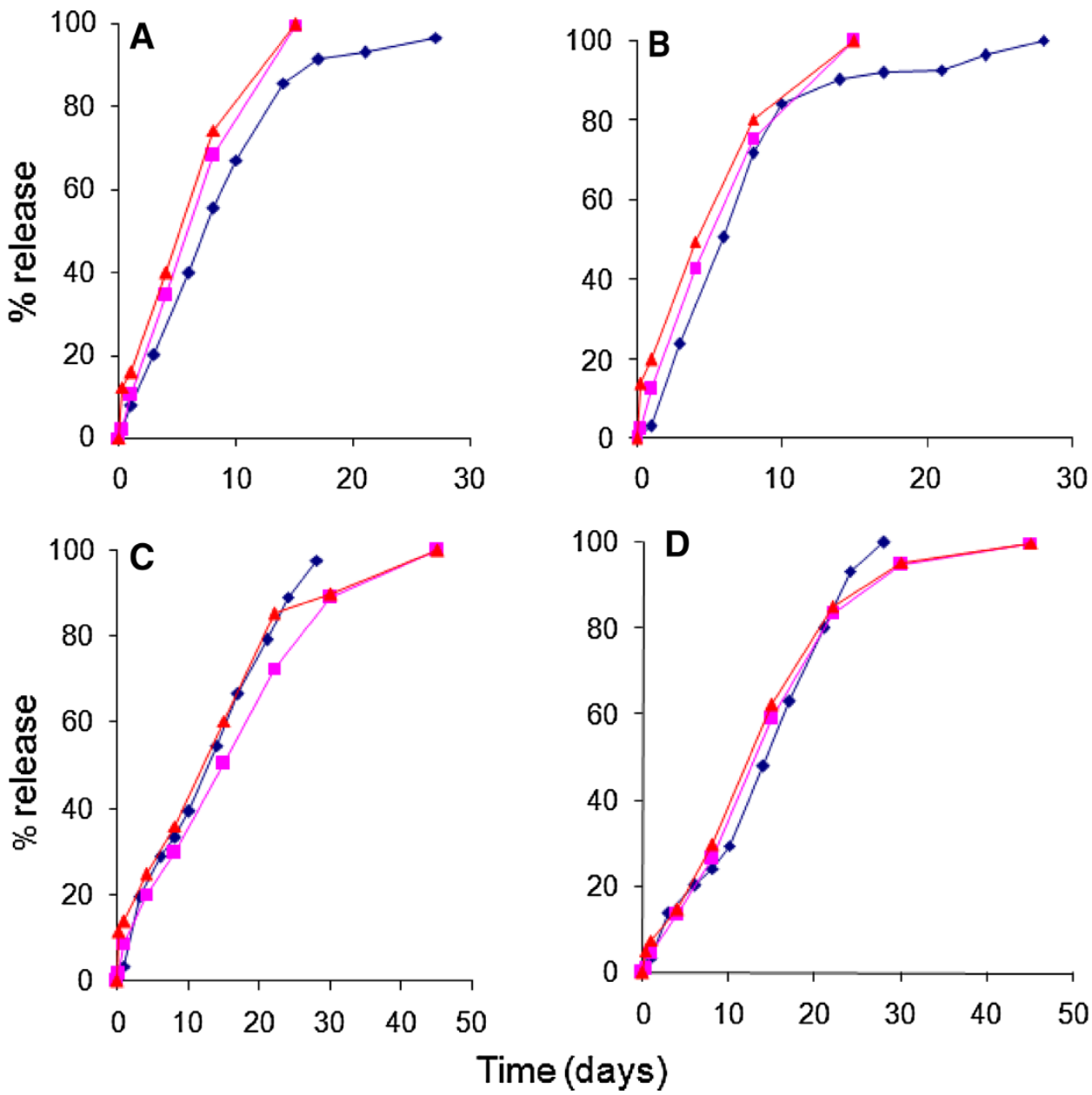
Fig. 6 Level A IVIVC for risperidone PLGA microspheres using Nelson-Wagner method

Fig. 7 Level A IVIVC for risperidone PLGA microspheres using fractional AUC profile
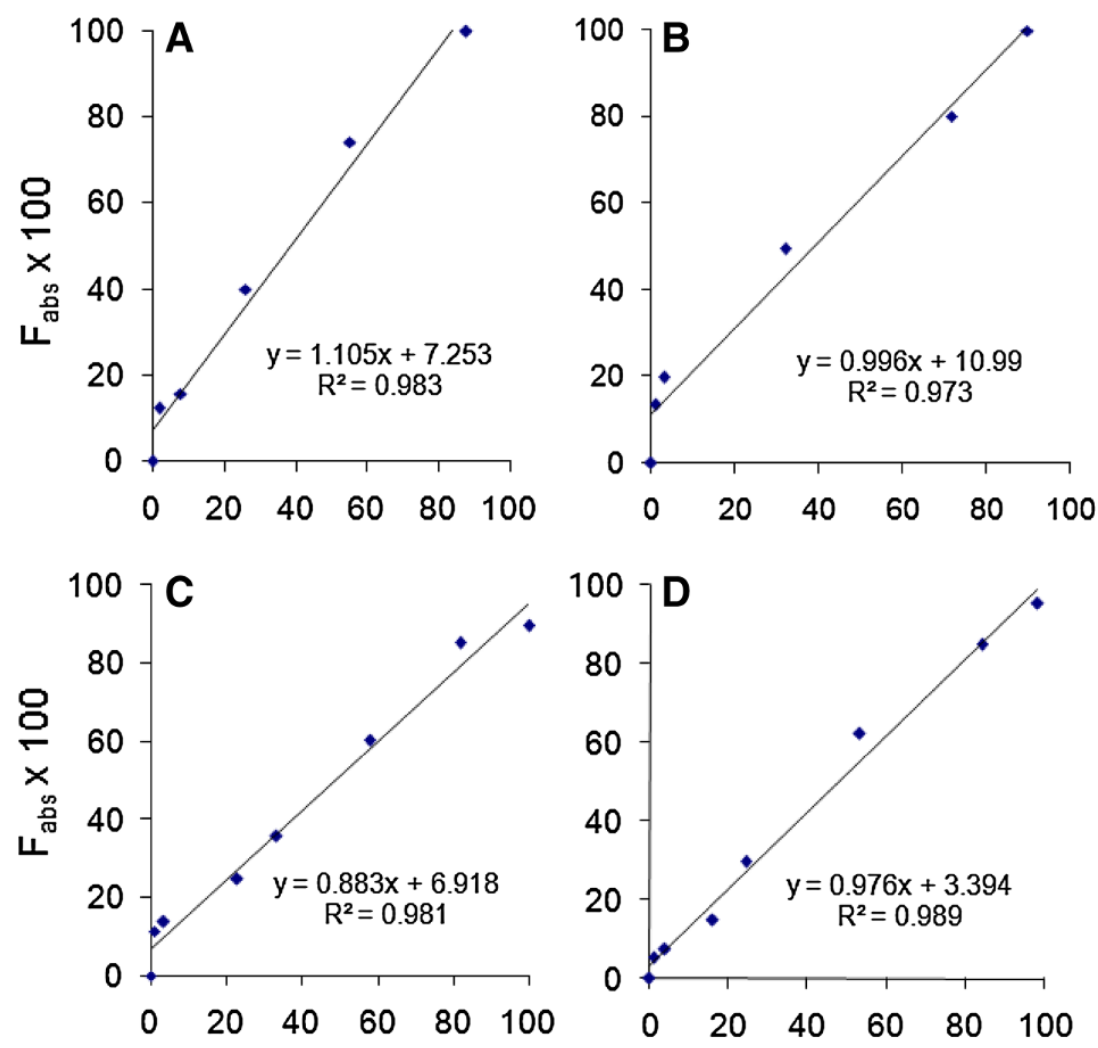

$\%$ in vitro release
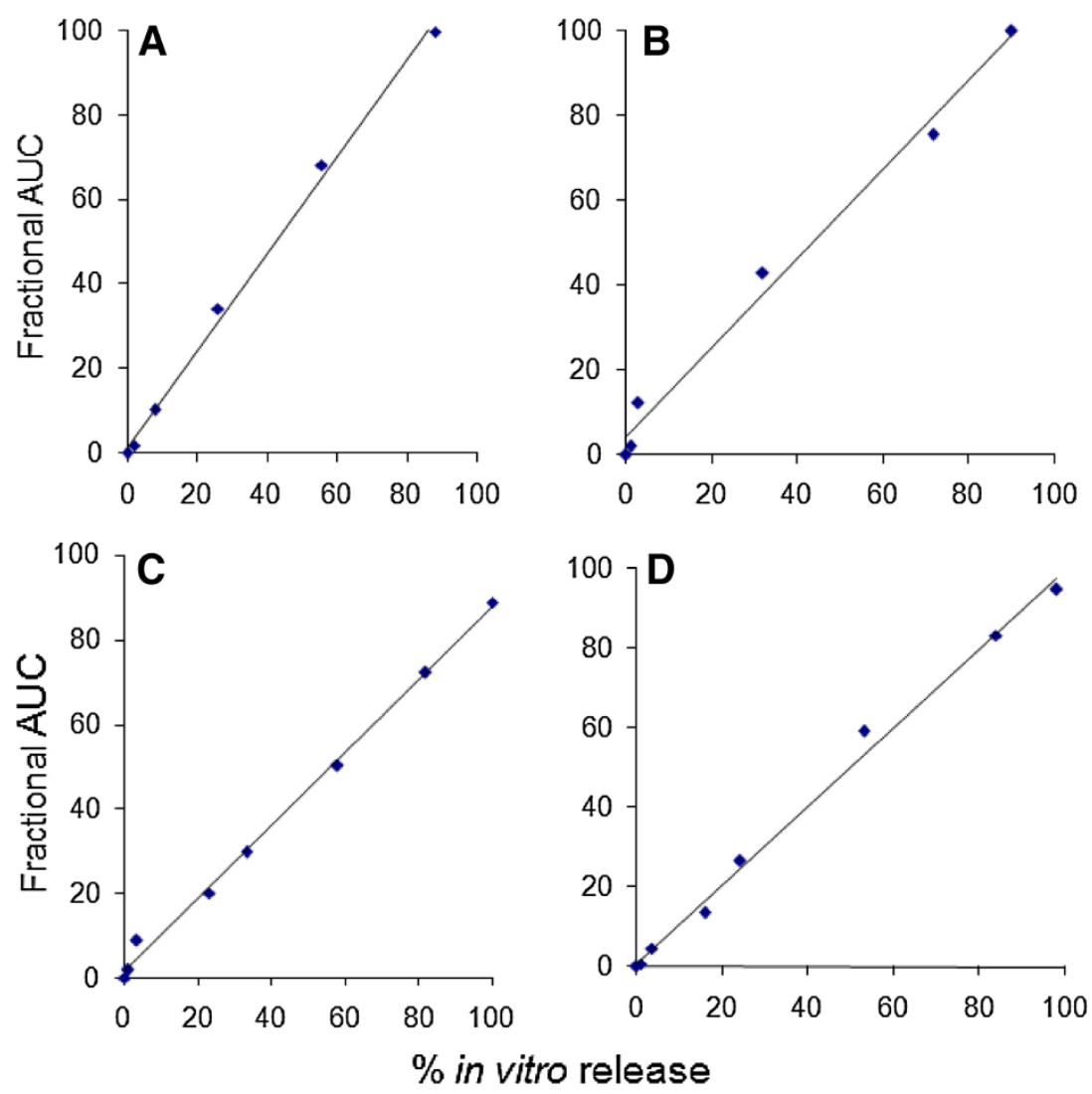
SUPAC-SS nonsterile semisolid dosage forms. scale-up and post-approval changes: chemistry, manufacturing, and controls; in vitro release testing and in vivo bioequivalence documentation 1997). For analysis using the NelsonWagner approach, the fraction absorbed (Fig. 3) was multiplied by 100 .

Figure 5 compares the plots of the fraction absorbed (i.e., $\left.F_{\text {abs }} \times 100\right)$ and fractional AUC with the in vitro data and highlights few noteworthy findings:

(a) Near superimposability of the release profiles was obtained in vitro (using the 'modified dialysis' method) with in vivo results. Similar findings were reported recently with another atypical antipsychotic, olanzapine, using the 'modified dialysis' method (D'Souza et al. 2014b).

(b) In vivo release, as measured by fractional AUC and fraction absorbed, is slightly faster than in vitro release. This phenomenon has been documented previously and attributed to the contribution of enzymes and foreign body response (Jiang et al. 2003). However, the shapes of the release profiles are essentially indistinct, and the time taken for nearly $90 \%$ drug release is very similar.

Figures 6, 7 and Table 1 describe the relationship between $\%$ in vitro release using the 'modified dialysis' method and the \% absorption, as calculated by the Nelson-Wagner method and fractional AUC. As can be clearly seen in the figures and the table, there was an excellent linear correlation $\left(R^{2}\right.$ values between 0.97 and $0.99, P>0.0001$ ) for the 50:50 (fast release) and 75:25 (slow release) formulations. The values of the slope range between 0.97 and 1.147, indicating that in vivo release occurred slightly faster than in vitro release for Formulations $A, B$ and $D$, while a slight lag was observed for Formulation $C$ (slope $=0.864-0.884)$. The values for the in vivo absorption models shown in Table 1 also attest to the suitability of using either approach to establishing an IVIVC. Indeed, the values obtained for the slope, intercept and $R^{2}$ are remarkably similar. Additionally, findings from the current study are in strong agreement with previous data where results from the fractional AUC approach have been comparable to the data generated using the Nelson-Wagner method (Chu et al. 2006; D'Souza et al. 2014b).

As recommended by the FDA guidance (FDA guidance for industry: extended-release oral dosage forms: development, evaluation and application of in vitro/in vivo correlations 1997), Figs. 8 and 9 depict the pooled IVIVCs for the 50:50 (fast release) and 75:25 (slow release) risperidone PLGA formulations. The results show an excellent Level A correlation between the in vitro data, obtained using the 'modified dialysis' method, and the in vivo
Table 1 IVIVC fit with in vivo absorption models

\begin{tabular}{llccl}
\hline Formulation & In vivo absorption model & Slope & Intercept & $R^{2}$ \\
\hline A & Fractional AUC & 1.147 & 1.401 & 0.996 \\
& Fraction absorbed & 1.105 & 7.253 & 0.983 \\
B & Fractional AUC & 1.050 & 4.190 & 0.989 \\
& Fraction absorbed & 0.996 & 10.99 & 0.974 \\
C & Fractional AUC & 0.864 & 1.534 & 0.999 \\
& Fraction absorbed & 0.884 & 6.917 & 0.981 \\
D & Fractional AUC & 0.989 & 0.821 & 0.995 \\
& Fraction absorbed & 0.976 & 3.395 & 0.990 \\
\hline
\end{tabular}

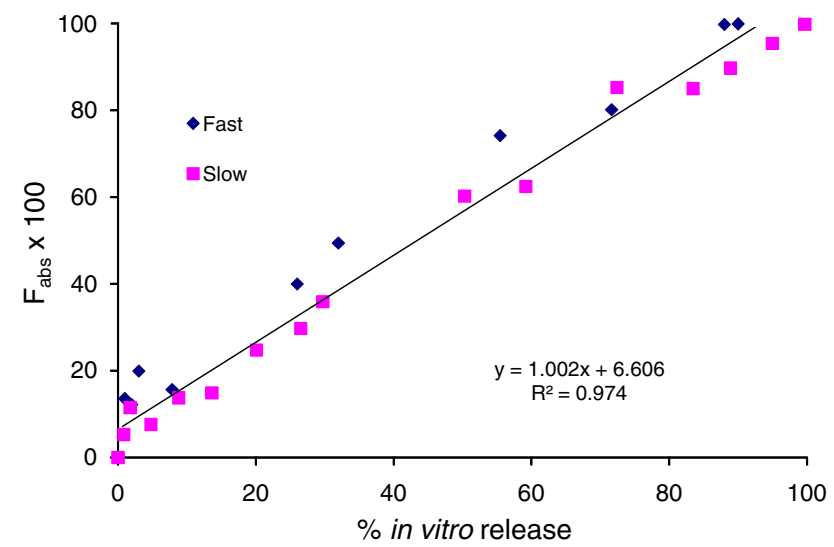

Fig. 8 Pooled IVIVC for risperidone PLGA microsphere formulations using Nelson-Wagner method (Level A)

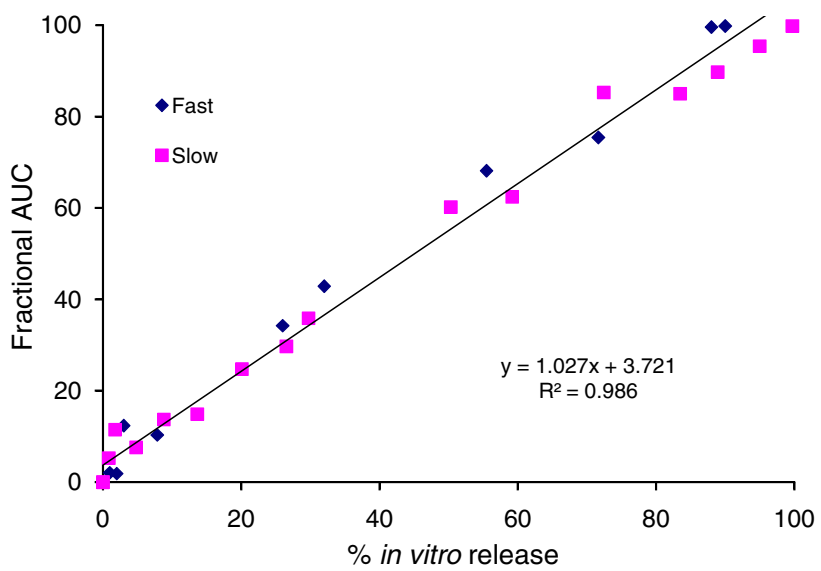

Fig. 9 Pooled IVIVC for risperidone PLGA microsphere formulations using fractional AUC profile (Level A)

results, using the fraction absorbed (Fig. 8) and the fractional AUC (Fig. 9) profiles. With both analyses, values of the slope were nearly equal to 1 , confirming that in vivo release for the slow and fast release formulations (50:50 and 75:25 PLGA) followed a similar trend, with minimal 
lag. The intercept values ranged between 3 and 6; with a higher value obtained with the Nelson-Wagner approach, as described in Sect. "Fraction absorbed". In summary, the results in Figs. 8 and 9 demonstrate an excellent Level A correlation $\left(R^{2}\right.$ value greater than 0.97$)$ between in vitro release of risperidone from PLGA microspheres and in vivo release. From literature, such type of correlation has not been previously reported with this molecule (Amann et al. 2010; Su et al. 2009).

While reports have cited the lack of standardized in vitro test methods as an important reason for dearth of an IVIVC or a lack of 1:1 correlation with parenteral microspheres (Rawat et al. 2012), data from the current study prove that with proper selection of an in vitro method, an IVIVC can be obtained. A similar finding and a near 1:1 correlation was also recently reported on olanzapine PLGA microspheres by our group (D'Souza et al. 2014b). Thus, results from the current study indicate that in vitro release using the 'modified dialysis' method is an excellent predictor of in vivo behavior of small molecules such as risperidone encapsulated into PLGA matrices and can be used as an indirect measure or surrogate for in vivo performance from developmental or clinical dosage forms.

\section{Conclusions}

A 'modified dialysis' method was selected to evaluate the in vitro behavior of risperidone PLGA microspheres formulated using 50:50 and 75:25 PLGA copolymers. This method was discriminatory and able to accurately distinguish the formulations on the basis of their release rates. In vitro release profiles exhibited a rank order similar to the results obtained in vivo by the FDA-recommended deconvolution approach (Nelson-Wagner method) or fractional AUC. Using both in vivo approaches, a near 1:1 linear Level A correlation between in vitro and in vivo release was obtained for the four formulations evaluated, suggesting that the 'modified dialysis' technique was suitable for in vitro release assessment of risperidone PLGA dosage forms.

Acknowledgments The research described in this manuscript was performed while the authors were affiliated with the University of Kentucky, Lexington, KY. The authors wish to thank Oakwood Labs, Oakwood, $\mathrm{OH}$, and the Graduate School, University of Kentucky, Lexington, KY, for their financial support.

Conflict of interest The authors state that there are no conflicts of interest.

Open Access This article is distributed under the terms of the Creative Commons Attribution License which permits any use, distribution, and reproduction in any medium, provided the original author(s) and the source are credited.

\section{References}

Amann LC, Gandal MJ, Lin R, Liang Y, Siegel SJ (2010) In vitroin vivo correlations of scalable PLGA-Risperidone implants for the treatment of schizophrenia. Pharm Res 27(8):1730-1737

Aubert-Pouessel A, Bibby DC, Venier-Julienne M-C, Hindre F, Benoit J-P (2002) A novel in vitro delivery system for assessing the biological integrity of protein upon release from PLGA microspheres. Pharm Res 19(7):1046-1051

Aubert-Pouessel A, Venier-Julienne M-C, Clavreul A, Sergent M, Jollivet C, Montero-Menei CN, Garcion E, Bibby DC, Menei P, Benoit J-P (2004) In vitro study of GDNF release from biodegradable PLGA microspheres. J Control Release 95(3):463-475

Bain DF, Munday DL, Smith A (1999) Modulation of rifampicin release from spray-dried microspheres using combinations of poly-(D, L-lactide). J Microencapsul 16(3):369-385

Blanco-Prieto MJ, Campanero MA, Besseghir K, Heimgatner F, Gander B (2004) Importance of single or blended polymer types for controlled in vitro release and plasma levels of a somatostatin analogue entrapped in PLA/PLGA microspheres. J Control Release 96(3):437-448

Capan Y, Woo BH, Gebrekidan S, Ahmed S, DeLuca PP (1999) Preparation and characterization of poly (D, L-lactide-co-glycolide) microspheres for controlled release of poly(L-lysine) complexed plasmid DNA. Pharm Res 16(4):509-513

Chaubal M (2002) Polylactides/glycolides-excipients for injectable drug delivery and beyond. Drug Deliv Technol 5(2):34-36

Chu D-F, Fu X-Q, Liu W-H, Liu K, Li Y-X (2006) Pharmacokinetics and in vitro and in vivo correlation of huperzine A loaded poly(lactic-co-glycolic acid) microspheres in dogs. Int J Pharm 325(1-2):116-123

DeLuca PP, Mehta RC, Hausberger AG, Thanoo BC (1993) Biodegradable polyesters for drug and polypeptide delivery. Polymer delivery systems, properties and applications

Diaz RV, Llabres M, Evora C (1999) One-month sustained release microspheres of ${ }^{125}$ I-bovine calcitonin: in vitro-in vivo studies. J Control Release 59(1):55-62

D’Souza SS, DeLuca PP (2006) Methods to assess in vitro drug release from injectable polymeric particulate systems. Pharm Res 23(3):460-474

D’Souza S, Faraj JA, DeLuca PP (2013a) Microsphere delivery of risperidone as an alternative to combination therapy. Eur $\mathbf{J}$ Pharm Biopharm 85(3):631-639

D’Souza S, Faraj JA, Giovagnoli S, DeLuca PP (2013b) Development of risperidone PLGA microspheres. J Drug Deliv 2013:620464

D’Souza S, Faraj JA, Giovagnoli S, DeLuca PP (2013c) Preparation, characterization and in vivo evaluation of olanzapine poly $(\mathrm{D}$, L-lactide-co-glycolide) (PLGA) microspheres. J Pharm 2013:831381

D’Souza S, Faraj JA, Dorati R, DeLuca PP (2014a) A short term quality control tool for biodegradable microspheres. AAPS Pharm Sci Tech 15(3):530-541

D'Souza S, Faraj JA, Giovagnoli S, DeLuca PP (2014b) IVIVC from long acting olanzapine microspheres. Int $\mathrm{J}$ Biomater 2014:407065

Ertl B, Platzer P, Wirth M, Gabor F (1999) Poly(d, 1-lactic-coglycolic acid) microspheres for sustained delivery and stabilization of camptothecin. J Control Release 61(3):305-317

FDA guidance for industry: extended release oral dosage forms: development, evaluation and application of in vitro/in vivo correlations (1997)

FDA guidance for industry: SUPAC-SS nonsterile semisolid dosage forms. Scale-up and post approval changes: chemistry, 
manufacturing, and controls; in vitro release testing and in vivo bioequivalence documentation (1997)

He J, Feng M, Zhou X, Ma S, Jiang Y, Wang Y, Zhang H (2011) Stabilization and encapsulation of recombinant human erythropoietin into PLGA microspheres using human serum albumin as a stabilizer. Int J Pharm 416(1):69-76

Heya T, Mikura Y, Nagai A, Miura Y, Futo T, Tomida Y, Shimizu H, Toguchi H (1994) Controlled release of thyrotropin releasing hormone from microspheres: evaluation of release profiles and pharmacokinetics after subcutaneous Administration. J Pharm Sci 83(6):798-801

Hora MS, Rana RK, Nunberg JH, Tice TR, Gilley RM, Hudson ME (1990) Release of human serum albumin from poly(lactide-coglycolide) microparticles. Pharm Res 7(11):1190-1194

Jiang G, Woo BH, Kang F, Singh J, DeLuca PP (2002) Assessment of protein release kinetics, stability and protein polymer interaction of lysozyme encapsulated poly(D, L,-lactide-co-glycolide) microspheres. J Control Release 79(1-3):137-145

Jiang G, Qiu W, DeLuca PP (2003) Preparation and in vitro/in vivo evaluation of insulin-loaded poly(acryloyl-hydroxyethyl starch)PLGA composite microspheres. Pharm Res 20(3):452-459

Kostanski JW, DeLuca PP (2000) A novel in vitro release technique for peptide-containing biodegradable microspheres. AAPS Pharm Sci Tech 1(1):4

Kostanski JW, Dani BA, Reynolds G-A, Bowers CY, DeLuca PP (2000) Evaluation of orntide microspheres in a rat animal model and correlation to in vitro release profiles. AAPS Pharm Sci Tech 1(4):27

Kwak HH, Shim WS, Son MK, Kim YJ, Kim TH, Youn HJ, Kang SH, Shim CK (2010) Efficacy of a new sustained-release microsphere formulation of exenatide, DA-3091, in Zucker diabetic fatty (ZDF) rats. Eur J Pharm Sci 12(40):2

Longo WE, Goldberg EP (1985) Hydrophilic albumin microspheres. Methods Enzymol 112:18-26

Martinez M, Rathbone M, Burgess D, Huynh M (2008) In vitro and in vivo considerations associated with parenteral sustained release products: a review based upon information presented and points expressed at the 2007 controlled release society annual meeting. J Control Release 129(2):79-87

Mehta RC, Jeyanthi R, Calis S, Thanoo BC, Burton KW, DeLuca PP (1994) Biodegradable microspheres as depot system for parenteral delivery of peptide drugs. J Control Release 29(3):375-384

Middleton JC, Tipton AJ (2000) Synthetic biodegradable polymers as orthopedic devices. Biomaterials 21(23):2335-2346

Morita T, Sakamura Y, Horikiri Y, Suzuki T, Yoshino H (2001) Evaluation of in vivo release characteristics of protein-loaded biodegradable microspheres in rats and severe combined immunodeficiency disease mice. J Control Release 73(2-3):213-221

Nastruzzi C, Esposito E, Cortesi R, Gambari R, Menegatti E (1993) Kinetics of bromocriptine release from microspheres: comparative analysis between different in vitro models. J Microencapsul 11(5):565-574

Park TG (1995) Degradation of poly(lactic-co-glycolic acid) microspheres: effect of copolymer composition. Biomaterials 16(15):1123-1130

Park TG, Lee HY, Nam YS (1998) A new preparation method for protein loaded poly(D, L-lactic-co-glycolic acid) microspheres and protein release mechanism study. J Control Release 55(2-3):181-191

Park EJ, Na DH, Lee KC (2007) In vitro release study of monoPEGylated growth hormone-releasing peptide-6 from PLGA microspheres. Int J Pharm 343(1-2):281-283

Rawat A, Stippler E, Shah VP, Burgess DJ (2011) Validation of USP apparatus 4 method for microsphere in vitro release testing using Risperdal ${ }^{\circledR}$ Consta $^{\circledR}$. Int J Pharm 420(2):198-205

Rawat A, Bhardwaj U, Burgess DJ (2012) Comparison of in vitroin vivo release of Risperdal ${ }^{\circledR}$ Consta ${ }^{\circledR}$ microspheres. Int J Pharm 434(1-2):115-121

Schrier J, DeLuca P (1999) Recombinant human bone morphogenetic protein-2 binding and incorporation in PLGA microsphere delivery systems. Pharm Dev Technol 4(4):611-621

Shmueli RB, Ohnaka M, Miki A, Pandey NB, Lima e Silva R, Koskimaki JE, Kim J, Popel AS, Campochiaro PA, Green JJ (2013) Long-term suppression of ocular neovascularization by intraocular injection of biodegradable polymeric particles containing a serpin-derived peptide. Biomaterials 34:30

Siewert M, Dressman J, Brown CK, Shah VP (2003) FIP/AAPS guidelines to dissolution/in vitro release testing of novel/special dosage forms. AAPS Pharm Sci Tech 4(1):7

Su Z, Sun F, Shi Y, Jiang C, Meng Q, Teng L, Li Y (2009) Effects of formulation parameters on encapsulation efficiency and release behavior of risperidone poly(D, L-lactide-co-glycolide) microsphere. Chem Pharm Bull 57(11):1251-1256

Sun L, Zhou S, Wang W, Li X, Wang J, Weng J (2009) Preparation and characterization of porous biodegradable microspheres used for controlled protein delivery. Colloids Surf A 345(1-3):173-181

Uppoor VRS (2001) Regulatory perspectives on in vitro (dissolution)/ in vivo (bioavailability) correlations. $J$ Control Release 72(1-3): 127-132

Wagenaar BW, Muller BW (1994) Piroxicam release from spraydried biodegradable microspheres. Biomaterials 15(1):49-54

Wagner JG, Nelson E (1963) Per cent absorbed time plots derived from blood level and/or urinary excretion data. J Pharm Sci 52(6):610-611

Washington C (1990) Drug release from microdisperse systems: a critical review. Int J Pharm 58(1):1-12

Wei G, Pettway GJ, McCauley LK, Ma PX (2004) The release profiles and bioactivity of parathyroid hormone from poly(lacticco-glycolic acid) microspheres. Biomaterials 25(2):345-352

Woo BH, Kostanski JW, Gebrekidan S, Dani BA, Thanoo BC, DeLuca PP (2001) Preparation, characterization and in vivo evaluation of 120-day poly(D, L-lactide) leuprolide microspheres. J Control Release 75(3):307-315

Xuan J, Lin Y, Huang J, Yuan F, Li X, Lu Y, Zhang H, Liu J, Sun Z, Zou H, Chen Y, Gao J, Zhong Y (2013) Exenatide-loaded PLGA microspheres with improved glycemic control: in vitro bioactivity and in vivo pharmacokinetic profiles after subcutaneous administration to SD rats. Peptides 46:172-179

Yen SY, Sung KC, Wang JJ, Yoa-Pu HuO (2001) Controlled release of nalbuphine propionate from biodegradable microspheres: in vitro and in vivo studies. Int J Pharm 220(1-2):91-99 